Relations industrielles

Industrial Relations

\title{
Alfred CHAPENTIER, Montée triomphale de la CTCC, un volume, 123 pages, Montréal, 1951. En vente chez l'auteur, 7080, rue Hutchison, Montréal, \$0.75.
}

\section{Gérard Dion}

Volume 7, numéro 1-2, décembre 1951, mars 1952

URI : https://id.erudit.org/iderudit/1023086ar

DOI : https://doi.org/10.7202/1023086ar

Aller au sommaire du numéro

Éditeur(s)

Département des relations industrielles de l’Université Laval

ISSN

0034-379X (imprimé)

1703-8138 (numérique)

Découvrir la revue

Citer ce compte rendu

Dion, G. (1951). Compte rendu de [Alfred CHAPENTIER, Montée triomphale de la CTCC, un volume, 123 pages, Montréal, 1951. En vente chez l'auteur, 7080, rue Hutchison, Montréal, \$0.75.] Relations industrielles / Industrial Relations, 7(1-2), 69-69. https://doi.org/10.7202/1023086ar

Tous droits réservés (C Département des relations industrielles de l’Université Laval, 1952
Ce document est protégé par la loi sur le droit d'auteur. L'utilisation des services d'Érudit (y compris la reproduction) est assujettie à sa politique d'utilisation que vous pouvez consulter en ligne.

https://apropos.erudit.org/fr/usagers/politique-dutilisation/ 
maine politique », attirera certainement l'attention d'une foule de nos gens que préoccupe cette activité du mouvement ouvrier même chez nous.

Pour terminer, nous pouvons simplement citer une phrase de la conclusion même de l'étude qui explique pourquoi ce travail a été fait: « Si nous avons entrepris cette tâche, c'est parce que le syndicalisme moderne a pris dans la société d'aujourd'hui une place de premiere importance, au point que dans la plupart des pays libres, il imprime sa marque sur l'évolution économique et sociale. Nous l'avons fait aussi parce que le syndicalisme se trouve en pleine mêlée. C'est un mouvement et une organisation qu'on exalte, qu'on subit ou qu'on combat. Tant chez les dirigeants que dans les autres classes, la conviction a fini par s'imposer, qu'un pays ne pouvait plus grand'chose, sans le syndicalisme, et à plus forte raison contre lui $\gg$.

Fernand Jolicoeur

Alfred Chapentien, Montée triomphale de la CTCC, un volume 123 pages. Montréal 1951. En vente chez l'auteur, 7080 , rue Hutchison, Montréal, \$0.75.

Deux heures de lecture suffisent à un lecteur au courant des faits pour parcourir ce que M. le Chanoine Pichette appelle le * Mémorial» de la Confédération des travailleurs catholiques du Canada. L'ancien président général de la CTCC, qui a vécu les trente années du développement de ce mouvement n'essaie pas de faire une véritable histoire, mais plutôt une chronologie des évènements importants qui ont marqué son développement. Ce livre sera utile pour ceux qui auront à écrire une histoire des travailleurs de la province de Québec. En attendant il est une excellente synthèse pour initier une personne qui veut avoir un aperçu du syndicalisme catholique chez nous. Il convient de féliciter l'auteur qui, on le sait, est un travailleur authentique par sa contribution à la littérature sociale dans le Québec.

\section{GÉrard Dion}

\section{SERvice D'Education DE LA C.T.C.C., Syndicat, instrument de libération des travailleurs,} une brochure 30 pages, Québec, 1951.

Brochure destinée à faire comprendre aux travailleurs la nécessité la nature, le rôle, le fonctionnement d'un syndicat ainsi que l'organisation et la fonction de la C.T.C.C.

Service d'Education, Pour bien diriger un syndicat, Service d'éducation du Conseil Général des Syndicats Catholiques de Québec, 1950, une brochure 76 pages.

Brochure à l'intention des officiers de syndicats contient ce qu'il faut savoir pour faire fonctionner démocratiquement un syndicat: procédure des assemblées, tenue des dossiers, comptabilité syndicale. Faite spécialement pour syndicats ouvriers, mais peut être utilisée par n'importe quel groupement.

Readings in Labor Economics, edited by Francis S. Doody. Associate Professor of Economics: Boston University, College of Business Administration - 1950 - Addison-Wesley Press, Inc., Cambridge, 42, Mass.

John W. McConnell and Robert F. Risley, Economic Security: A Study of Communitu Needs and Resources, 79 pp., New York State School of Industrial and Labor Relations; Cornell University - Bulletin No. 18, Ithaca, N.Y., 1951.

C.H. Nonthcotr, M.A., PH.D., The Personnel Aspects of Management, Institute of Personnel Management, Management House, Hill Street, London, W.I., Prix 3s. Od. 